\title{
Ambition and Action
}

\section{By Florence Egal, Formerly Nutrition Officer, UN Food and Agriculture Organization}

In the context of renewed global momentum for improving nutrition, and as it embarks with FAO on leading the implementation of the UN resolution for a Decade of Action on Nutrition, WHO has recently published its nutrition strategy Ambition and Action in Nutrition 2016-2025. This strategy was developed in the context of the internal WHO reform in the first-ever strategic exercise on nutrition undertaken by WHO.

It recognizes malnutrition as an intersectoral topic and therefore the need for an integrated perspective. It therefore promotes a twin track approach: further coordination on nutrition within WHO on the one hand, and increased intersectoral and interinstitutional collaboration on the other. It recalls the history of WHO in nutrition and sets out the rationale for internal change

Similar efforts have been and are being carried out by other UN agencies and are indeed essential to the success of the Decade. Each agency has its mandate, entry points and history and has a comparative advantage in contributing to success, in collaboration with partners. But FAO and WHO, as the two UN specialized agencies on nutrition, are expected to provide leadership and guidance in their respective sectors and to work together. This requires agencies to revisit their role, identify key actors within the organization at national, regional and global levels, redefine tasks, and allocate resources. This corporate understanding and ownership of an expanded strategy needs to go hand in hand with redefined collaboration mechanisms at all levels.

This document is therefore timely and certainly constitutes a milestone for WHO at a time of management change. It will also open new doors and entry points for interagency and intersectoral collaboration, and contribute to awareness-raising and policy 
convergence both within the health sector and within the framework of the 2030 Sustainable Development Agenda.

WHO certainly has a major role to play - and related responsibility - in advancing nutrition and it should definitely provide leadership, guidance and monitoring within the health sector. And health staff have a key advocacy role with other sectors to incorporate nutrition in relevant strategies, policies and projects.

This being said, some statements and grey areas would deserve further clarification to avoid confusion and misunderstandings. It is unlikely that WHO leadership will be acceptable beyond the health sector. At a time of increased awareness of the complex intersectoral and inter-institutional nature of nutrition, it is urgent to work towards a common framework within which each actor will find recognition and be able to contribute to commonly agreed goals. Inter-agency collaboration on nutrition, until now limited to the nutrition units of each agency--which had for historical and institutional reasons very different functions--has been a major challenge. Bringing on board other units within these agencies as well as other agencies should help to overcome this challenge and allow exploration of further mechanisms of collaboration. The accent should be put on synergy rather than leadership, on contributing or facilitating rather than fostering or steering, and on adding value to initiatives and processes led by other sectors. And nutrition policies should not be limited to engaging with nutrition actors, but also with other actors that can impact on nutrition, making them accountable for achieving this impact.

The UN resolution "calls upon FAO and WHO to lead the implementation of the Decade of Action on Nutrition in collaboration with the World Food Programme (WFP), the International Fund for Agricultural Development (IFAD) and the United Nations Children's Fund (UNICEF), and involving coordination mechanisms such as the United Nations System Standing Committee on Nutrition (UNSCN) and multi-stakeholder platforms such as the Committee on World Food Security (CFS)". Ways forward for these interactions could have been developed further. 
The reference to CFS ("that concerns mostly ministers of agriculture and other food security actors, including the food industry”) in particular could be misleading and should be corrected ${ }^{1}$. The Private Sector Mechanism of the CFS brings together private enterprises right across the agri-food value chain, from farmers, to input providers, cooperatives, processors, and small and medium-sized enterprises (SMEs) and food companies; it is therefore not limited to food industry and it is important that all actors be given appropriate attention and recognition. Given their key role in the CFS, it is also surprising that there is no mention of the Civil Society Mechanism "which brings together representatives of smallholder farmers, pastoralists, fisherfolks, indigenous peoples, agricultural and food workers, landless, women, youth, consumers, urban food insecure and NGOs”. The CFS - which reports to the UN General Assembly through the Economic and Social Council (ECOSOC)--provides an inclusive international and intergovernmental platform to develop and endorse policy recommendations and guidance to ensure food security and nutrition for all.

Nutrition is indeed as an "enabler" of several SDGs but the association actually goes both ways, as SDGs are also a pre-requisite to good nutrition. Nutrition can also contribute to linking the SDGs, and therefore to further integration of the 2030 Agenda for Sustainable Development. More attention should therefore have been given to local level governance where action will eventually take place and to sustainability (including of Essential Nutrition Actions, and on their impact on local development).

In this perspective, the references to sustainable diets in the document are most welcome, since this concept can provide the basis to reorient dysfunctional food systems ${ }^{2}$ from a demand rather than supply/value chain entry point: informed consumers and public procurement are playing a lead role. However, these references are often limited to

1

http://www.fao.org/fileadmin/templates/cfs/Docs1516/About/CFS Multistakehol der Approach.pdf

2

http://glopan.org/sites/default/files/documents/ForesightReportExecSummary.pd $\mathrm{f}$ 
quality, safety and environmental dimensions, too often ignoring social aspects. Efforts to improve diets could be enriched through collaboration with FAO and other relevant institutions. The same would of course be true of references to agriculture and food systems (including on climate change), which fall within the mandate of FAO and are central to the work of the CFS since 2013.

Effective collaboration of WHO and FAO to assist member-states in joining forces at local level remains the major challenge - and opportunity - to operationalize the UN Decade on Nutrition and complement the work carried out at global and national level. Joint leadership of the two UN specialized agencies is essential to facilitating policy development and action to address all forms of malnutrition and ensuring convergence, engagement and accountability of actors (including donors) at all levels. 